Original research article

\title{
Moonlight human ribosomal protein L13a downregulation is associated with p53 and HER2/neu expression in breast cancer
}

\author{
Ghader Molavi 1, 2, Nasser Samadi 1, 2, 3, Shahriar Hashemzadeh ${ }^{4,5}$, Monireh Halimi ${ }^{6 *}$, \\ Elaheh Zadeh Hosseingholi ${ }^{7}$ \\ ${ }^{1}$ Tabriz University of Medical Sciences, Drug Applied Research Center, Tabriz, Iran \\ 2 Tabriz University of Medical Sciences, Faculty of Advanced Medical Sciences, Department of Molecular Medicine, Tabriz, Iran \\ ${ }^{3}$ Tabriz University of Medical Sciences, Faculty of Medicine, Department of Biochemistry, Tabriz, Iran \\ ${ }^{4}$ Tabriz University of Medical Sciences, Tuberculosis and Lung Disease Research Center, Tabriz, Iran \\ ${ }^{5}$ Tabriz University of Medical Sciences, Imam Reza Hospital, General and Vascular Surgery Department, Tabriz, Iran \\ ${ }^{6}$ Tabriz University of Medical Sciences, School of Medicine, Department of Pathology, Tabriz, Iran \\ ${ }^{7}$ Azarbaijan Shahid Madani University, Faculty of Basic Sciences, Department of Biology, Tabriz, Iran
}

\begin{abstract}
Breast cancer is the most common malignancy among females worldwide. Recent studies have shown extra-ribosomal roles of the moonlight ribosomal proteins in the development of human cancers. Accurate quantification of the gene expression level is based on the selection of the reference genes whose expression is independent of cancer properties and patient's characteristics. The aim of this study was the evaluation of the expression level of a previously proposed ribosomal protein as moonlight, L13a (RPL13A), in breast cancer samples and their adjacent tissues. Its association with genes of known roles in developing cancers was also investigated. Traditionally used housekeeping genes were selected and their expression was analyzed in 80 surgically excised breast tissue specimens (40 tumors and 40 tumor-adjacent tissues) by applying three software tools including GeNorm, NormFinder, and BestKeeper to select the most stable reference genes. Then, mRNA expression levels of RPL13A and p53 were evaluated. Additionally, protein expression levels of RPL13A were measured. It was demonstrated that PUM1 and ACTB are the most reliable reference genes and RPL13A is the least stable gene. There was a positive correlation between RPL13A and p53 mRNA expression levels in all the tumor samples. Moreover, significant downregulation of RPL13A expression levels was revealed in HER2+ tumor samples compared to HER2- ones. There was also a marked decrease in $p 53$ mRNA expression levels in HER2+ tumor subtypes. Our results suggest that there is a probable relationship between RPL13A decreased expression with p53 and HER2/neu expression in the breast cancer.
\end{abstract}

Keywords: Breast neoplasms; ErbB receptors; Ribosomal proteins; Tumor suppressor protein p53

\section{Highlights:}

- RPL13A was least stable gene reference gene in breast cancer samples.

- Positive correlation between RPL13A and p53 mRNA expression levels was detected in all studied tumor samples.

- Significant downregulation of RPL13A expression levels was revealed in HER2+ tumor samples compared to HER2- tumor samples.

- p53 mRNA expression levels showed considerable decrease in HER2+ tumor subtypes.

\begin{abstract}
Abbreviations:
rRNAs, ribosomal ribonucleic acids; RPs, ribosomal proteins; RPL13A, human ribosomal; protein L13a; GAIT, interferon-gammaactivated inhibitor of translation; cDNA, complementary DNA; qRT-PCR, quantitative reverse transcription PCR;

GAPDH, glyceraldehyde 3-phosphate dehydrogenase; ACTB, actin $\beta$; B2M, beta-2-microglobulin; PUM1, pumilio RNA binding family member 1; Ct, threshold cycle; IHC, Immunohistochemical; PBS, phosphate-buffered saline; HRP, horseradish peroxidase; $\mathrm{DAB}, 3$, 3-diaminobenzidine tetrahydrochloride; HER2+, human epidermal growth factor receptor 2 positive; TCGA, The Cancer Genome Atlas; IRESs, internal ribosome entry sites; oxLDL, oxidized low-density lipoprotein
\end{abstract}

\footnotetext{
* Author for correspondence: Monireh Halimi, Tabriz University of Medical Sciences, School of Medicine, Department of Pathology, Tabriz 5166614733, Iran; e-mail: halimimonireh@gmail.com http://doi.org/10.32725/jab.2020.008

Submitted: 2020-02-27 • Accepted: 2020-05-28 • Prepublished online: 2020-06-17

J Appl Biomed 18/2-3: 46-53 • EISSN 1214-0287 • ISSN 1214-021X

(c) 2020 The Authors. Published by University of South Bohemia in České Budějovice, Faculty of Health and Social Sciences.

This is an open access article under the CC BY-NC-ND license.
} 


\section{Introduction}

Breast cancer has a high prevalence among females (Bray et al., 2018). The complexity of the prognosis, diagnosis, and treatment of this disease originates from the biological heterogeneity of neoplastic breast epithelial cells, which in turn is caused by genomics, transcriptomics, proteomics and epigenetic diversities (Cava et al., 2015). However, early-stage diagnosis of breast cancer results in a more effective response to treatment, improved survival prospects, and reduced risk of metastasis and mortality (Cheng and Ueno, 2012).

The eukaryotic complex structure of the ribosome is essential for protein biosynthesis in the cell. This organelle is made up of ribosomal ribonucleic acids (rRNAs) and ribosomal proteins (RPs). The integrated 80S ribosome consists of two large (60S) and small (40S) subunits (Bhavsar et al., 2010). Due to large variations in the type of proteins and the rate of protein synthesis in different cells, tissue-specific expression of ribosomal components has been reported in normal human tissues (Buszczak et al., 2014). Moreover, it was shown that specific ribosomal protein genes are expressed differently in some pathological conditions such as cancer and cardiovascular diseases (Wang et al., 2015). Contrary to the general assumption, RPs independent of their main involvement in the protein biosynthesis and ribosome biogenesis can also participate in important cellular functions including DNA replication and repair, cell proliferation and differentiation, and apoptosis (Xu et al., 2016). These multifunctional RPs are called moonlight ribosomal proteins. By revealing the function of RPs in multiple extra-ribosomal activities, the role of these proteins as diagnostic and/or prognostic markers has been demonstrated in cancer patients (Molavi et al., 2019). Notably, human ribosomal protein L13a (RPL13A) is a component of the 60S ribosomal subunit. However, it does not have a proven role in the protein synthesis, assembly of mature $80 \mathrm{~S}$ ribosomes, and rRNA processing. This molecule is a moonlight ribosomal protein in the mature ribosome compartment with the release capacity from the ribosomal subunit in the phosphorylated state. The phosphorylated RPL13A blocks the translation of interferon-gamma-activated inhibitor of translation (GAIT) element-containing target mRNAs following the IFN- $\gamma$-induced signaling (Mukhopadhyay et al., 2008; 2009). To understand how molecular alterations drive cancer, recent studies have focused on the detection of expression differences between cancerous and normal tissues (Pierouli et al., 2019).

This study aimed to evaluate the expression of RPL13A in both mRNA and protein levels in tumors and related adjacent tissues of breast cancer patients. We also measured P53 mRNA expression and its association with RPL13A in these patients. Furthermore, stability of this gene was compared to the stability of other common housekeeping genes by applying three different software tools.

\section{Material and methods}

\section{Collection of human breast cancer specimens}

A total of 40 fresh breast tumors and the paired adjacent tissues were obtained from newly diagnosed breast cancer patients and were confirmed with an oncologist. These specimens were recruited from Valiasr Hospitals, Tabriz, Iran between 2018 and 2019. Table 1 shows the clinicopathological information of the participants. Notably, the patients were not treated with chemotherapeutic agents at the time of sampling. Fresh samples were divided into two segments, one of which was used for pathology analysis, whereas the second was collected in liquid nitrogen and stored frozen at $-80^{\circ} \mathrm{C}$.

Table 1. Characteristics of transitional breast cancer samples

\begin{tabular}{lc}
\hline Characteristic & Patients, $n$ \\
\hline Total number of patients & 40 \\
Age & \\
$\quad$ Median (range) & $45(21-65)$ \\
Histological grade & \\
1 & 10 \\
2 & 22 \\
3 & 8 \\
Tumor type & \\
ER+ & 16 \\
ER- & 24 \\
HER2+ & 12 \\
HER2- & 28 \\
\hline
\end{tabular}

\section{Evaluation of the mRNA expression level of RPL13A and 553}

\section{Total RNA isolation and cDNA synthesis}

The preserved tumor and tumor-adjacent samples were cut into the smallest possible pieces and total RNA was isolated using TRIzol reagent (Invitrogen; Thermo Fisher Scientific, Inc.) by mechanical homogenizing and according to the manufacturer's protocol. Then, the isolated RNA was purified using the Phenol/Chloroform extraction method (Toni et al., 2018). For complementary DNA (cDNA) synthesis, PrimeScript RT Master Mix (Takara Bio, Inc.) was applied in the retro-transcription reaction mixture.

\section{Real-time PCR}

Gene expression is modulated by different intra- and extracellular factors. Therefore, identification of gene expression modification via common methods such as real-time quantitative reverse transcription PCR ( $\mathrm{qRT}-\mathrm{PCR}$ ) requires normalization of the target-genes expression levels to the expression of the housekeeping gene with minimum variation in a wide variety of conditions. To date, some genes such as glyceraldehyde 3-phosphate dehydrogenase (GAPDH) and actin $\beta$ (ACTB) have been used as the general-purpose reference genes (Kasai et al., 2003). Despite their wide-spread nature, the variability of known reference genes in human cancers was shown (Jo et al., 2019). Since RPL13A is a conventionally used reference gene for the normalization of the gene expression in different cells and tissues, its reliability as a reference gene was validated in this study. Then, mRNA expression levels of RPL13A and $p 53$ were evaluated relative to the most stable reference genes. For this purpose, one microliter of RT products was used as a template for the real-time $P C R$ reaction with the SYBR Premix Ex Taq ${ }^{\mathrm{TM}}$ II (TaKaRa, \#RR820A/B, Japan) reagent on the LightCycler 96 System (Roche Diagnostics, Mannheim, Germany). Table 2 presents sequences of forward and reverse primers used in $P C R$ reactions to amplify $p 53$ and five candidate reference genes, including $A C T B$, beta-2-microglobulin (B2M), GAPDH, RPL13A, and pumilio RNA binding family member 1 (PUM1). Each reaction was carried out with three technical replicates. 
Table 2. Characteristics of the selected genes and their primers

\begin{tabular}{|c|c|}
\hline Gene & Primers \\
\hline GAPDH & $\begin{array}{l}\text { F 5'-TCTCCTCTGACTTCAACAGCGA-3' } \\
\text { R 5'-CCCTGTTGCTGTAGCCAAATTC-3' }\end{array}$ \\
\hline ACTB & $\begin{array}{l}\text { F 5‘-TCACAATGTGGCCGAGGACTTT-3‘ } \\
\text { R 5‘-AGAAGTGGGGTGGCTTTTAGGATG-3‘ }\end{array}$ \\
\hline RPL13A & $\begin{array}{l}\text { F 5'-GCAAAGATCCATTACCGGAAG-3‘ } \\
\text { R 5'-ACAGTCTTTATTGGGTTCACAC-3‘ }\end{array}$ \\
\hline$B 2 M$ & 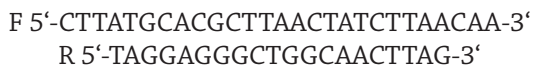 \\
\hline PUM1 & $\begin{array}{l}\text { F 5'-AGTGGGGGACTAGGCGTTAG-3“' } \\
\text { R 5'-GTTTTCATCACTGTCTGCATCC-3، }\end{array}$ \\
\hline$p 53$ & $\begin{array}{l}\text { F 5'-TCAACAAGATGTTTTGCCAACTG-3' } \\
\text { R 5'-ATGTGCTGTGACTGCTTGTAGATG-3' }\end{array}$ \\
\hline
\end{tabular}

\section{Data analysis}

To determine the most stable reference genes, qRT-PCR data related to the housekeeping genes were analyzed using three Excel-based widely applied software tools, GeNorm (Vandesompele et al., 2002), NormFinder (Andersen et al., 2004), and BestKeeper (Pfaffl et al., 2004). GeNorm sorts the tested reference genes according to $M$ value. $M$ value or gene expression normalization factor is an average pair-wise variation of a single candidate with all others. The higher the $M$ value revealed the less stability of the selected gene expression (Vandesompele et al., 2002). NormFinder identifies optimal reference genes by the combination of the estimated intra- and inter-group variations for each candidate reference gene, called stability value (Andersen et al., 2004). BestKeeper helps users to determine the best reference genes using pair-wise correlation analysis of candidate reference genes (Pfaffl et al., 2004). Finally, the two most stable genes were used as the internal controls to normalize the expression of RPL13A and p53 using the 2- $\Delta \Delta \mathrm{Ct}$ method (Livak and Schmittgen, 2001).

\section{Evaluation of the protein expression level of RPL13A}

\section{Immunohistochemical staining}

All tissue samples were subjected to the Immunohistochemical (IHC) process to determine the expression levels of RPL13A. Briefly, tissues were fixed in $20 \%$ buffered formalin and then embedded in paraffin to prepare the blocks of tissues. Then, the blocks were sectioned ( $4 \mu \mathrm{m}$ thickness) and placed on the glass slides. Afterward, the slides were put aside at $60^{\circ} \mathrm{C}$ overnight, de-paraffinized in xylene, and subsequently rehydrated by ethanol series. Besides, $3 \% \mathrm{H}_{2} \mathrm{O}_{2}$ in methanol was used to quench endogenous peroxidase activity of samples. The tissue sections were subjected to heating in the sodium citrate buffer at $\mathrm{pH} 6.0$ for $30 \mathrm{~min}$ to retrieve antigen. After a triple round of washing with phosphate-buffered saline (PBS, $\mathrm{pH}$ 7.4), the tissue sections were incubated with rabbit polyclonal anti-RPL13A antibody (Abcam Inc.) at $4{ }^{\circ} \mathrm{C}$ overnight followed by incubation with anti-rabbit secondary antibody conjugated with horseradish peroxidase (HRP) (Dako, EnVision system, Denmark) at room temperature for $45 \mathrm{~min}$. After washing with PBS solution, 3, 3-diaminobenzidine tetrahydrochloride (DAB) $/ \mathrm{H}_{2} \mathrm{O}_{2}$ solution was applied for about 5 min to visualize the peroxidase activity. For the next stage, the sections were washed with water, and then $0.1 \%$ hematoxylin was used for counterstaining. Finally, after cover slipping the slides with a rapid mounting medium (Entellan, Merck, Germany), they were analyzed under a light microscope. Negative controls were obtained by omitting the primary antibodies from the staining procedure (Jeddi et al., 2018).

\section{Immunohistochemical analysis}

The expression level of RPL13A was evaluated based on a scoring system, in which the staining intensity was scored from 0 to 3 (no staining $=0$, weak $=1$, moderate $=2$, and strong $=3$ ). Also, according to the percentage of the stained cells relative to all epithelial cells, the expression was scored as $(0,<5 \% ; 1$, $6-10 \%$; 2, 11-50\%; and 3, >51\%). The expression index was calculated by adding the two individual scores (Kasai et al., 2003). Then, the specimens were graded from 0 to 3 based on the total scores, i.e. the score of 0 as negative staining (-), 1-4 as weakly positive (1+), 5-8 as moderately positive (2+), and $9-12$ as strongly positive $(3+)$. For statistical analysis, the negative and weakly positive groups ( - and $1+$ ) were recorded as negative, while the moderately and strongly positive groups $(2+$ and $3+)$ were defined as positive.

\section{Results}

\section{Expression levels of candidate reference genes}

To assess the $C t$ values of all five candidate reference genes in the samples, qPCR method was used. mRNA expression levels of candidate genes displaying median $\mathrm{Ct}$ values ranging from 16.99 (GAPDH) to 28.04 (B2M) are shown in Fig. 1.

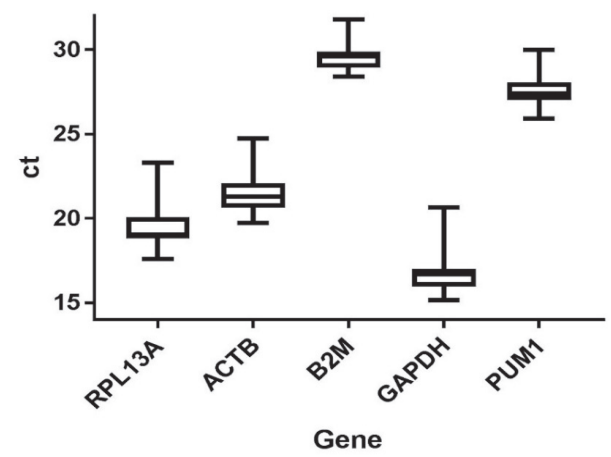

Fig. 1. Ct values of the candidate reference genes in 80 breast tissue specimens

The selected reference genes were further analyzed in tumor and tumor-adjacent with the software programs of GeNorm, NormFinder, and BestKeeper (Tables 3 and 4). GeNorm calculates the stability of the expression according to the $M$ value. As shown in Table 3, the genes with the smallest $M$ value were PUM1 and ACTB (1.07), which were the most stable genes in all samples in the present study. However, the least stable gene was RPL13A with a high $\mathrm{M}$ value of 1.34 . The results of the NormFinder analysis, which evaluates the expression stability of each candidate independently, are shown in Table 3. Consistent with the results of GeNorm, PUM1 was ranked the first in the list of the most stable reference genes, and RPL13A was the most variable reference gene compared to the other candidates. The analysis results of BestKeeper, which evaluates the stability of the reference gene expression using raw $\mathrm{Cq}$ values, are given in Table 4 . The results showed that covariance and standard deviation values of B2M and RPL13A were the lowest and the highest of the reference genes, respectively. 
Table 3. Ranking of housekeeping genes by GeNorm and NormFinder

\begin{tabular}{|c|c|c|c|c|c|}
\hline \multirow[t]{2}{*}{ Candidate genes } & \multicolumn{2}{|c|}{ GeNorm } & \multicolumn{3}{|c|}{ NormFinder } \\
\hline & Rank & M value $^{\mathrm{a}}$ & Rank & $\mathrm{SD}^{\mathrm{b}}$ & Acc. $S^{c}$ \\
\hline PUM1 & 1,2 & 1.07 & 1 & 0.7117 & 0.7117 \\
\hline ACTB & 1,2 & 1.07 & 2 & 0.8157 & 0.5413 \\
\hline GAPDH & 3 & 1.20 & 3 & 0.9092 & 0.4712 \\
\hline$B 2 M$ & 4 & 1.22 & 4 & 1.017 & 0.435 \\
\hline RPL13A & 5 & 1.34 & 5 & 1.263 & 0.4304 \\
\hline
\end{tabular}

${ }^{a}$ stability factor of expression; ${ }^{b}$ standard deviation (stability value); ${ }^{c}$ accumulated standard deviation.

Table 4. Results of five housekeeping genes by BestKeeper

\begin{tabular}{lcccccccc}
\hline Gene & \multicolumn{3}{c}{ Ct } & \multicolumn{3}{c}{ x-fold } \\
\cline { 2 - 9 } & GM $^{\mathrm{a}}$ & Min & Max & SD $^{\text {b }}$ & \%CV & Min & Max & SD $^{c}$ \\
\hline B2M & 29.54 & 28.41 & 31.78 & 0.65 & 2.19 & -2.19 & 4.73 & 1.57 \\
PUM1 & 27.49 & 25.92 & 29.99 & 0.71 & 2.58 & -2.97 & 5.66 & 1.64 \\
ACTB & 21.44 & 19.74 & 24.75 & 0.92 & 4.28 & -3.25 & 9.92 & 1.89 \\
GAPDH & 16.8 & 15.16 & 20.66 & 0.92 & 5.46 & -3.12 & 14.51 \\
RPL13A & 19.52 & 17.59 & 23.31 & 1.08 & 5.51 & -3.80 & 13.87 & 2.11 \\
\hline
\end{tabular}

${ }^{a}$ stability factor of expression; ${ }^{b}$ standard deviation (stability value); ${ }^{c}$ accumulated standard deviation.

Considering the differences among the ranking results of the three used algorithms, a method was used to calculate the final ranking of the reference genes (Chen et al., 2011). The ranking of each reference gene in all the algorithms was listed and then the geometric means of the three ranking numbers were calculated. The smallest geometric mean shows the most stable genes. As shown in Table 5, PUM1 and ACTB were determined as the most reliable reference genes.

Table 5. Final ranking of five candidate reference genes in all samples

\begin{tabular}{lcccc}
\hline Ranking & geNorm & $\begin{array}{c}\text { Norm } \\
\text { Finder }\end{array}$ & BestKeeper & Overall \\
\hline 1 & PUM1-ACTB & PUM1 & B2M & PUM1 \\
2 & PUM1-ACTB & ACTB & PUM1 & ACTB \\
3 & GAPDH & GAPDH & ACTB-GAPDH & B2M \\
4 & B2M & B2M & ACTB-GAPDH & GAPDH \\
5 & RPL13A & RPL13A & RPL13A & RPL13A \\
\hline
\end{tabular}

\section{mRNA expression levels of RPL13A and $p 53$}

According to the research design, quantitative-PCR was performed to evaluate the RPL13A and $p 53$ mRNA expression in all tissue samples. As can be seen in Fig. 2 and 3, RPL13A and p53 expression levels were significantly down-regulated in the tumor tissues compared to the related tumor-adjacent tissues $(p<0.05)$ in human epidermal growth factor receptor 2 positive (HER2+) subtype samples. Moreover, a decrease of the RPL13A expression in the tumor of breast cancer subtypes of luminal-like and basal-like was observed in comparison to their adjacent samples, but their reduction $p$-values were not significant $(p>0.05)$. In addition, the decrease of $p 53$ expression levels in the luminal-like subtype tumor samples and its increase in the basal-like subtype samples were observed, but the $p$-values of their expression changes were not significant $(p>0.05)$. Finally, it was found that a positive correlation exists between expression levels of RPL13A and p53 in the breast cancer tissue samples (Fig. 4).

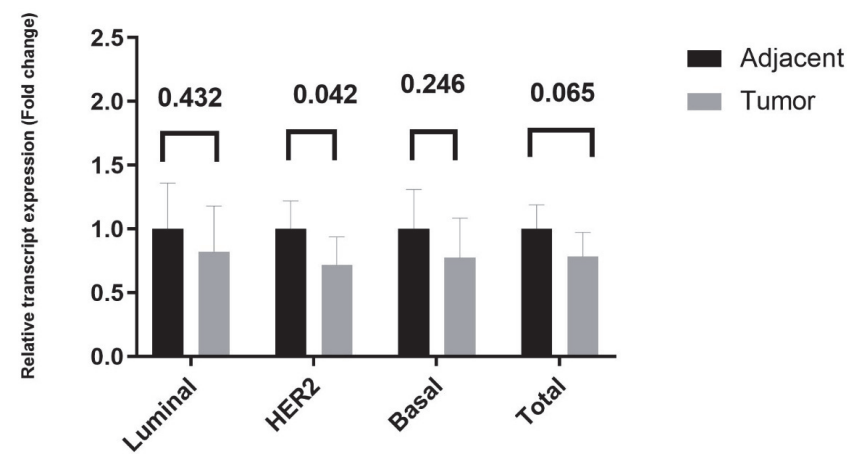

Fig. 2. Quantitative PCR analysis of mRNA expression levels of RPL13A in different subtypes of samples

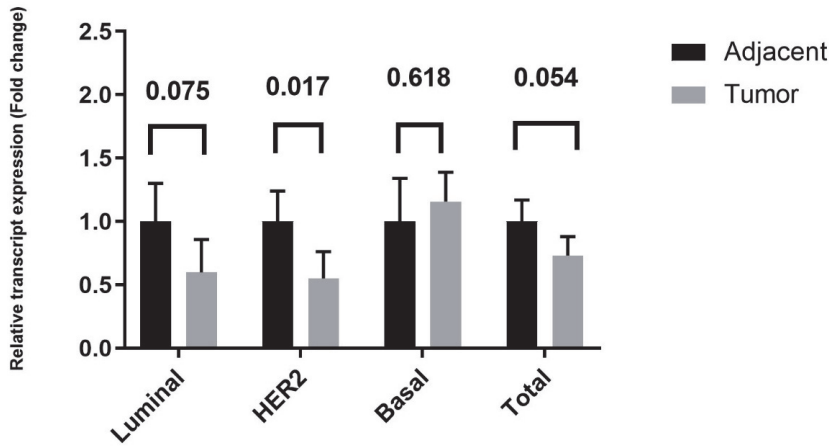

Fig. 3. Quantitative PCR analysis of mRNA expression levels of $p 53$ expression in different subtypes of samples 


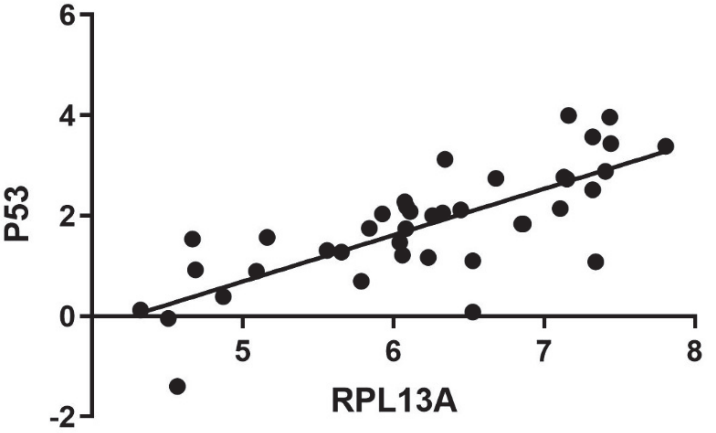

Fig. 4. Pearson correlation analyses of the relationship between mRNA expression levels of RPL13A and p53 in breast cancer tissue samples
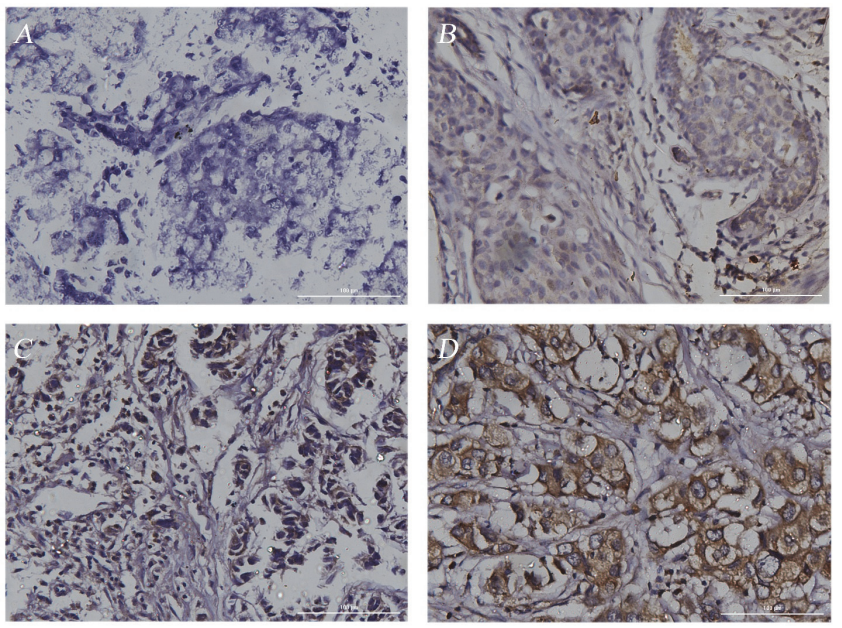

Fig. 5. Immunohistochemical staining of RPL13A expression. (A) Negative staining; (B) Weakly positive and (C) Moderately positive; and (D) Strongly positive staining of RPL13A in breast cancer tissues. Scale bars $=100 \mu \mathrm{m}$.

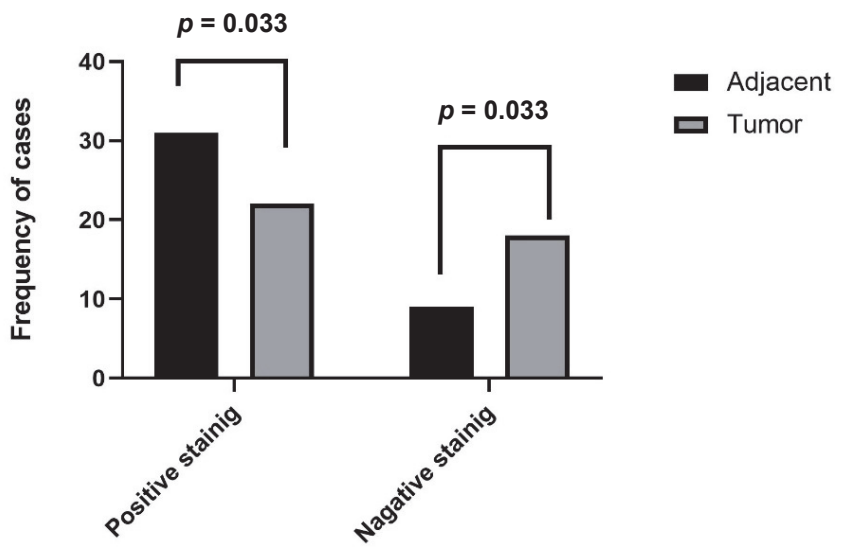

Fig. 6. Immunohistochemical analysis of expression - RPL13A protein expression was significantly down-regulated in the tumor tissues compared to the adjacent tissues $(p<0.05)$

\section{Protein expression levels of RPL13A}

We determined the protein level of RPL13A by immunohistochemical staining in the collected tumor and tumor-adjacent tissues. Our results showed that RPL13A is predominantly expressed in the cytoplasm of the breast carcinoma cells (Fig. 5). It was also found that (Fig. 6) the positive staining rate of RPL13A expression was $27.5 \%(22 / 80)$ in tumor tissues, which was significantly $(p<0.05)$ lower than that in the tumor-adjacent tissues $38.5 \%$ (31/80).

\section{Association between RPL13A expression and clinicopathological criteria}

Table 6 summarizes the correlation analyses between the clinicopathological criteria and the mRNA expression levels of RPL13A. According to the table, no significant association was observed with age, tumor size, and histological grade. The decreased expression level of RPL13A was markedly associated with HER2 expression status of tumors $(p=0.023)$. Notably, the same results were obtained when correlation analysis was performed between clinicopathological criteria and protein expression levels of RPL13A (Table 7).

Table 6. Association between clinicopathological criteria and mRNA expression of RPL13A

\begin{tabular}{lcc}
\hline $\begin{array}{l}\text { Clinicopathological } \\
\text { criteria }\end{array}$ & $\begin{array}{c}R P L 13 A \text { expression } \\
\text { (Mean } \pm \text { SEM) }\end{array}$ & $P$ value (T-test) \\
\hline $\begin{array}{l}\text { Age } \\
\quad 45 \text { years }\end{array}$ & $-6.180 \pm 0.204$ & 0.425 \\
$\quad<45$ years & $-5.945 \pm 0.206$ & \\
Tumor size & & \\
$\leq 5 \mathrm{~cm}$ & $-6.160 \pm 0.177$ & 0.574 \\
$>5 \mathrm{~cm}$ & $-5.994 \pm 0.240$ & \\
Histological grade & & \\
1 & $-6.016 \pm 0.160$ & \\
2 & $-5.956 \pm 0.240$ & \\
3 & $-6.443 \pm 0.277$ & \\
Tumor type & & \\
ER+ & & \\
ER- & $-5.899 \pm 0.245$ & \\
HER+ & $-6.181 \pm 0.177$ & 0.346 \\
HER- & $-5.859 \pm 0.176$ & $\mathbf{0 . 0 2 5}$ \\
\hline
\end{tabular}

The bold characters denote that the $p$ value is less than 0.05 , which has the statistical significance.

\section{Discussion}

It is commonly agreed that breast cancer is the second cause of death in women throughout the world. Besides, genetic and epigenetic diversities of individuals have been considered as the main causes of clinical and morphological heterogeneity of breast cancer tumors. Notably, one of the main properties of cancer are the complex signaling pathways. Therefore, identification and control of the genes, whose dysregulation is involved in the tumorigenesis, is a main challenge of investigators worldwide. Therefore, the aim of the present study was the evaluation of the expression of one of the ribosomal proteins, RPL13A, whose extra-ribosomal functions in translational control of the genes involved in cancer have been verified recently. The schematic representation of the methodology and obtained results in this research are given in Fig. 7. 
Table 7. Association between clinicopathological criteria and protein expression of RPL13A

\begin{tabular}{|c|c|c|c|c|}
\hline Clinicopathological criteria & No. of patients (\%) & Positive $n(\%)=18(45)$ & Negative $n(\%)=22(55)$ & $P$ value (T-test) \\
\hline \multicolumn{5}{|l|}{ Age } \\
\hline$\geq 45$ years & 21 & $10(47.6)$ & $11(52.4)$ & \multirow[t]{2}{*}{0.726} \\
\hline$<45$ years & 19 & $8(42.1)$ & $11(57.9)$ & \\
\hline \multicolumn{5}{|l|}{ Tumor size } \\
\hline$\leq 5 \mathrm{~cm}$ & 18 & $9(50)$ & $9(50)$ & \multirow[t]{2}{*}{0.565} \\
\hline$>5 \mathrm{~cm}$ & 22 & $9(40.9)$ & 13(59.1) & \\
\hline \multicolumn{5}{|l|}{ Histological grade } \\
\hline 1 & 10 & $5(50)$ & $5(50)$ & \multirow{3}{*}{0.848} \\
\hline 2 & 22 & $9(40.9)$ & $13(59.1)$ & \\
\hline 3 & 8 & $4(50)$ & $4(50)$ & \\
\hline \multicolumn{5}{|l|}{ Tumor type } \\
\hline ER+ & 16 & $6(37.5)$ & $10(62.5)$ & \multirow{3}{*}{0.436} \\
\hline ER- & 24 & $12(50)$ & $12(50)$ & \\
\hline HER + & 12 & $9(75)$ & $3(25)$ & \\
\hline HER- & 28 & $9(32.1)$ & $19(67.9)$ & 0.013 \\
\hline
\end{tabular}

The bold characters denote that the $p$ value is less than 0.05 , which has the statistical significance.

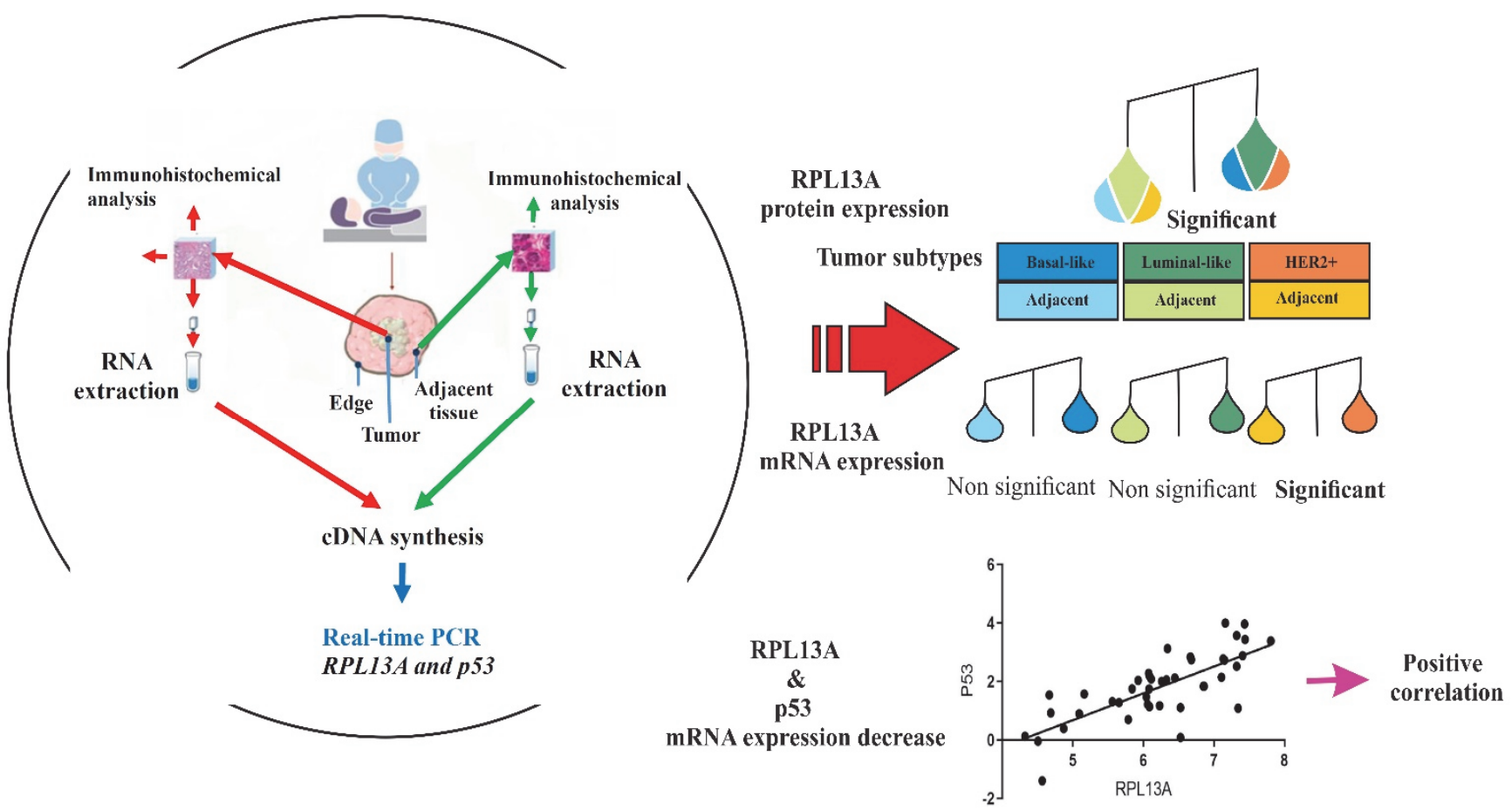

Fig. 7. Schematic representation of the methodology and obtained results in this research

RT-qPCR is one of the most frequently used methods for gene expression profiling. However, for the following reasons, the selection of reliable reference genes is the prerequisite of this study: (1) Data obtained from qRT-PCR should be normalized with appropriate reference genes. (2) The traditionally used reference genes are not necessarily suitable for normalization of the gene expression in human cancer research. (3) RPL13A is conventionally considered as a housekeeping gene in many studies. Two of the ideal reference gene criteria are: (1) its abundance in the studied tissues and (2) a low expression variation in the investigated samples. In this study, according to the analyses of three software tools (geNorm, NormFinder, and BestKeeper), the final ranking of the most reliable reference genes was indicated as follows: PUM1 > ACTB $>B 2 M>G A P D H>R P L 13 A$.
Thus, PUM1 and ACTB were the most reliable reference genes. This result was in parallel with the results obtained from the analysis of large-scale cancer gene expression datasets from the Cancer Genome Atlas (TCGA) database, which validated PUM1 and ACTB genes among the top stable reference genes (Jo et al., 2019). Also, the pan-cancer analysis of TCGA Data revealed PUM1 as the most suitable reference gene for qPCR normalization of expression data related to breast cancer (Krasnov et al., 2019). Moreover, the suitability of PUM1 as a reference gene was demonstrated in another largescale screening of the potential candidate reference genes from RNA-seq data. Notably, the large expression variation of RPL13A was also shown in the same study (Tilli et al., 2016). Contrary to the results of this study, the results of another study about the selection of the housekeeping genes as the 
references for the normalization of quantitative PCR data in breast cancer indicated the combination of PUM1/RPL13A as suitable reference genes (Kılıç et al., 2014). Such a condition may be due to the difference in the tumor subtypes samples used in these studies.

Fig. 2 shows that the mRNA expression level of RPL13A in tumor samples is weaker than the level in the tumor-adjacent samples, which is significant $(p<0.05)$ only in the comparison of HER2 + tumor samples with their adjacent samples. RPL13A mRNA expression decrease was reported in breast cancer through an investigation of the expression of $\sim 10,000 \mathrm{hu}-$ man tumors and over 700 normal tissues from TCGA (Dolezal et al., 2018). Additionally, RPL13A expression decrease was reported in pancreatic and cervical cancer tumors compared to normal tissues (Crnogorac-Jurcevic et al., 2001; Rajkumar et al., 2011). Interestingly, the increased level of the expression of RPL13A was shown in breast cancer and ovarian cancer patients following chemotherapy (Clarke et al., 2004; Modlich et al., 2004). Moreover, the decrease of RPL13A mRNA expression in $\mathrm{H} 605$ breast cancer cells, which were derived from HER2/neu transgenic mice, was previously reported (Chen et al., 2010).

According to the results presented in Fig. 4, there is a positive correlation between the RPL13A and p53 expression levels. It was demonstrated that some mRNAs, including p27, and p53 contain internal ribosome entry sites (IRESs) in their 5'untranslated region (5'-UTR), which mediate cap-independent translation initiation of these mRNAs. It was found that methylation posttranslational modification of rRNA increases the translation via IRES. Moreover, RPL13A causes methylation of rRNA and efficient translation via IRES elements of these mRNAs (Chaudhuri et al., 2007; King et al., 2010). However, as shown by the results of Fig. 3, there is a significant reduction in p53 expression levels in HER2+ tumor subtypes. Here, it is emphasized that previous studies verified the inverse relationship of p53 and p27 expressions with HER2 expression (Le et al., 2003; Payandeh et al., 2016). Therefore, the inverse relationship of RPL13A with HER2 expression may be mediated by p53 and p27. As can be seen in Tables 6 and 7 , there is a significant reduction in RPL13A expression levels in the HER2+ tumor samples compared to the HER2- samples with a $p$-value lower than that of the HER2+ tumor samples with their adjacent samples (Fig. 2). Therefore, it can be expected that HER2+ tumor-adjacent cells may express fewer levels of HER2 compared to tumor cells (Margan et al., 2016; Zubor et al., 2015).

According to gene-expression profiling, the major subtypes of breast cancer are HER2+, luminal-like, and basal-like (Perou et al., 2000). The luminal-like subtype is ER low or high and HER2 ${ }^{\text {low }}$. The basal-like subtype is ER- and HER2- (Malhotra et al., 2010). The results of Fig. 3 showed insignificantly decreased expression of p53 in this subtype. The inverse expression of p53 with the increased ERa expression in luminal tumors has been shown previously (Bado et al., 2018). As seen in Fig. 5, the expression level of RPL13A protein was significantly decreased in total tumor tissues compared to their adjacent tissues. It should be mentioned that the weaker expression of this ribosomal protein was shown in the results of a study, which investigated the expression differences of ribosomal proteins in the human normal and neoplastic colorectum (Kasai et al., 2003). However, the difference in the significance of RPL13A reduction in mRNA and protein levels in total samples (Fig. 2 and 6) may be derived from the posttranslational expression changes of RPL13A. GAPDH could function as a chaperone, protecting RPL13A from proteasomal degradation. Moreover, it has been shown that this shielding activity is lost following cell treatment with oxidized low-density lipoprotein (oxLDL) (Jia et al., 2012). According to the findings, breast cancer patients exhibited higher levels of oxLDL (Cedó et al., 2019).

\section{Conclusions}

The selection of suitable reference genes is important for seeking novel markers in studies of breast cancer in the future. The results of the present study demonstrated that moonlight ribosomal protein RPL13A is not always the outstanding reference gene for normalization of gene expression in the breast cancer samples. The results also proposed a probable relationship between RPL13A decreased expression with p53 and HER2/neu expression in the breast cancer. Since HER-2/neu overexpression is a poor prognostic factor for breast cancer patients, it is worthy to confirm the results of this study by including larger sample numbers and finding exact interaction pathways in future studies.

\section{Ethics approval and consent to participate}

The study was approved by the Ethics Committee of Tabriz University of Medical Sciences (Ethical code: TBZmed. REC.1397.022). Informed consent was taken from all participants.

\section{Human and animal rights}

No animals were used in this study. All reported experiments on humans were followed in accordance with the ethical standards of the committee responsible for human experimentation (institutional national), and with the Helsinki Declaration of 1975, as revised in 2013.

\section{Consent for publication}

Informed consent was obtained from each participant.

\section{Funding}

This work was financially supported by the grant (58873) from Tabriz University of Medical Sciences.

\section{Conflict of interests}

The authors declare that there is no conflict of interests regarding the authorship of the presented manuscript.

\section{References}

Andersen CL, Jensen JL, Orntoft TF (2004). Normalization of real-time quantitative reverse transcription-PCR data: a modelbased variance estimation approach to identify genes suited for normalization, applied to bladder and colon cancer data sets. Cancer Res 64(15): 5245-5250. DOI: 10.1158/0008-5472.CAN04-0496.

Bado I, Pham E, Soibam B, Nikolos F, Gustafsson JA, Thomas C (2018). Er $\beta$ alters the chemosensitivity of luminal breast cancer cells by regulating p53 function. Oncotarget 9(32): 22509-22522. DOI: 10.18632/oncotarget.25147.

Bhavsar RB, Makley LN, Tsonis PA (2010). The other lives of ribosomal proteins. Hum Genomics 4(5): 327-344. DOI: 10.1186/1479-7364-4-5-327.

Bray F, Ferlay J, Soerjomataram I, Siegel RL, Torre LA, Jemal A (2018). Global cancer statistics 2018: GLOBOCAN estimates of incidence and mortality worldwide for 36 cancers in 185 countries. CA Cancer J Clin 68(6): 394-424. DOI: 10.3322/caac.21492. 
Buszczak M, Signer RAJ, Morrison SJ (2014). Cellular differences in protein synthesis regulate tissue homeostasis. Cell 159(2): 242-251. DOI: 10.1016/j.cell.2014.09.016.

Cava C, Bertoli G, Castiglioni I (2015). Integrating genetics and epigenetics in breast cancer: Biological insights, experimental, computational methods and therapeutic potential. BMC Syst Biol 9(62). DOI: 10.1186/s12918-015-0211-x.

Cedó L, Reddy ST, Mato E, Blanco-Vaca F (2019). HDL and LDL: potential new players in breast cancer development. J Clin Med 8(6): 853. DOI: $10.3390 / \mathrm{jcm} 8060853$.

Chaudhuri S, Vyas K, Kapasi P, Komar AA, Dinman JD, Barik S, Mazumder B (2007). Human ribosomal protein L13a is dispensable for canonical ribosome function but indispensable for efficient rRNA methylation. RNA 13(12): 2224-2237. DOI: 10.1261/rna.694007.

Chen D, Pan X, Xiao P, Farwell MA, Zhang B (2011). Evaluation and identification of reliable reference genes for pharmacogenomics, toxicogenomics, and small RNA expression analysis. J Cell Physiol 226(10): 2469-2477. DOI: 10.1002/jcp.22725.

Chen H, Pimienta G, Gu Y, Sun X, Hu J, Kim MS, et al. (2010). Proteomic characterization of Her2/neu-overexpressing breast cancer cells. Proteomics 10(21): 3800-3810. DOI: 10.1002/ pmic.201000297.

Cheng YC, Ueno NT (2012). Improvement of survival and prospect of cure in patients with metastatic breast cancer. Breast cancer 19(3): 191-199. DOI: 10.1007/s12282-011-0276-3.

Clarke PA, Pestell KE, Stefano F Di, Workman P, Walton MI. (2004). Characterisation of molecular events following cisplatin treatment of two curable ovarian cancer models: contrasting role for p53 induction and apoptosis in vivo. Br J Cancer 91(8): 1614-1623. DOI: $10.1038 /$ sj.bjc.6602167.

Crnogorac-Jurcevic T, Efthimiou E, Capelli P, Blaveri E, Baron A, Terris B, et al. (2001). Gene expression profiles of pancreatic cancer and stromal desmoplasia. Oncogene 20(50): 7437-7446. DOI: 10.1038/sj.onc.1204935.

Dolezal JM, Dash AP, Prochownik EV (2018). Diagnostic and prognostic implications of ribosomal protein transcript expression patterns in human cancers. BMC cancer 18:275. DOI: 10.1186/ s12885-018-4178-z.

Jeddi F, Soozangar N, Sadeghi MR, Somi MH, Shirmohamadi M, Eftekhar-Sadat AT, Samadi N (2018). Nrf2 overexpression is associated with P-glycoprotein upregulation in gastric cancer. Biomed Pharmacother 97: 286-292. DOI: 10.1016/j. biopha.2017.10.129.

Jia J, Arif A, Willard B, Smith JD, Stuehr DJ, Hazen SL, Fox PL (2012). Protection of extraribosomal RPL13a by GAPDH and dysregulation by S-nitrosylation. Mol Cell 47(4): 656-663. DOI: 10.1016/j.molcel.2012.06.006.

Jo J, Choi S, Oh J, Lee SG, Choi SY, Kim KK, Park C (2019). Conventionally used reference genes are not outstanding for normalization of gene expression in human cancer research. BMC bioinformatics 20(10): 245. DOI: 10.1186/s12859-019-2809-2.

Kasai H, Nadano D, Hidaka E, Higuchi K, Kawakubo M, Sato TA, Nakayama J (2003). Differential expression of ribosomal proteins in human normal and neoplastic colorectum. J Histochem Cytochem 51(5): 567-573. DOI: 10.1177/002215540305100502.

Kılıç Y, Çelebiler AÇ, Sakızlı M (2014). Selecting housekeeping genes as references for the normalization of quantitative PCR data in breast cancer. Clin Transl Oncol 16(2): 184-190. DOI: 10.1007/ s12094-013-1058-5.

King HA, Cobbold LC, Willis AE (2010). The role of IRES trans-acting factors in regulating translation initiation. Biochem Soc Trans 38(6): 1581-1586. DOI: 10.1042/BST0381581.

Krasnov GS, Kudryavtseva AV, Snezhkina AV, Lakunina VA, Beniaminov AD, Melnikova NV, Dmitriev AA (2019). Pan-cancer analysis of TCGA data revealed promising reference genes for qPCR normalization. Front Genet 10: 97. DOI: 10.3389/ fgene.2019.00097.

Le XF, Claret FX, Lammayot A, Tian L, Deshpande D, LaPushin R, Tari AM, Bast RC (2003). The role of cyclin-dependent kinase inhibitor p27Kip1 in anti-HER2 antibody-induced G1 cell cycle arrest and tumor growth inhibition. J Biol Chem 278(26): 23441-23450. DOI: 10.1074/jbc.M300848200.
Livak KJ, Schmittgen TD (2001). Analysis of relative gene expression data using real-time quantitative PCR and the 2- ${ }^{\triangle \triangle C T}$ method. Methods 25(4): 402-408. DOI: 10.1006/meth.2001.1262.

Malhotra GK, Zhao X, Band H, Band V (2010). Histological, molecular and functional subtypes of breast cancers. Cancer Biol Ther 10(10): 955-960. DOI: 10.4161/cbt.10.10.13879.

Margan MM, Jitariu AA, Cimpean AM, Nica C, Raica M (2016). Molecular portrait of the normal human breast tissue and its influence on breast carcinogenesis. J Breast Cancer 19(2): 99-111. DOI: 10.4048/jbc.2016.19.2.99.

Modlich O, Prisack HB, Munnes M, Audretsch W, Bojar H (2004). Immediate gene expression changes after the first course of neoadjuvant chemotherapy in patients with primary breast cancer disease. Clin Cancer Res 10(19): 6418-6431. DOI: 10.1158/10780432.CCR-04-1031.

Molavi G, Samadi N, Hosseingholi EZ (2019). The roles of moonlight ribosomal proteins in the development of human cancers. J Cell Physiol. 234(6): 8327-8341. DOI: 10.1002/jcp.27722.

Mukhopadhyay R, Jia J, Arif A, Ray PS, Fox PL (2009). The GAIT system: a gatekeeper of inflammatory gene expression. Trends Biochem Sci 34(7): 324-331. DOI: 10.1016/j.tibs.2009.03.004.

Mukhopadhyay R, Ray PS, Arif A, Brady AK, Kinter M, Fox PL (2008). DAPK-ZIPK-L13a axis constitutes a negative-feedback module regulating inflammatory gene expression. Mol Cell 32(3): 371-382. DOI: 10.1016/j.molcel.2008.09.019.

Payandeh M, Shahriari-Ahmadi A, Sadeghi M, Sadeghi E. (2016). Correlations between HER2 expression and other prognostic factors in breast cancer: inverse relations with the Ki-67 index and P53 status. Asian Pac J Cancer Prev 17(3): 1015-1018. DOI: 10.7314/apjcp.2016.17.3.1015.

Perou CM, Sørlie T, Eisen MB, van de Rijn M, Jeffrey SS, Rees CA, et al. (2000). Molecular portraits of human breast tumours. Nature 406(6797): 747-752. DOI: 10.1038/35021093.

Pfaffl MW, Tichopad A, Prgomet C, Neuvians TP (2004). Determination of stable housekeeping genes, differentially regulated target genes and sample integrity: BestKeeper - Excelbased tool using pair-wise correlations. Biotechnol Lett 26(6): 509-515. DOI: 10.1023/B:BILE.0000019559.84305.47.

Pierouli K, Mitsis T, Papakonstantinou E, Vlachakis D (2019). Introductory Chapter: Gene Profiling in Cancer in the Era of Metagenomics and Precision Medicine. In: Gene Expression Profiling in Cancer. London: IntechOpen.

Rajkumar T, Sabitha K, Vijayalakshmi N, Shirley S, Bose MV, Gopal G, Selvaluxmy G (2011). Identification and validation of genes involved in cervical tumourigenesis. BMC Cancer 11(1): 80. DOI: 10.1186/1471-2407-11-80.

Tilli TM, da Silva Castro C, Tuszynski JA, Carels N (2016). A strategy to identify housekeeping genes suitable for analysis in breast cancer diseases. BMC Genomics 17(1): 639. DOI: 10.1186/s12864016-2946-1.

Toni LS, Garcia AM, Jeffrey DA, Jiang X, Stauffer BL, Miyamoto SD, Sucharov CC (2018). Optimization of phenol-chloroform RNA extraction. MethodsX 5: 599-608. DOI: 10.1016/j. mex.2018.05.011.

Vandesompele J, De Preter K, Pattyn F, Poppe B, Van Roy N, De Paepe A, Speleman F (2002). Accurate normalization of real-time quantitative RT-PCR data by geometric averaging of multiple internal control genes. Genome Biol 3(7): research0034.1. DOI: 10.1186/gb-2002-3-7-research0034.

Wang W, Nag S, Zhang X, Wang MH, Wang H, Zhou J, Zhang R (2015). Ribosomal proteins and human diseases: pathogenesis, molecular mechanisms, and therapeutic implications. Med Res Rev 35(2): 225-285. DOI: 10.1002/med.21327.

Xu X, Xiong X, Sun Y (2016). The role of ribosomal proteins in the regulation of cell proliferation, tumorigenesis, and genomic integrity. Sci China Life Sci 59(7): 656-672. DOI: 10.1007/ s11427-016-0018-0.

Zubor P, Hatok J, Moricova P, Kapustova I, Kajo K, Mendelova A, Kmetova Sivonova M, Danko J (2015). Gene expression profiling of histologically normal breast tissue in females with human epidermal growth factor receptor 2-positive breast cancer. Mol Med Rep 11(2): 1421-1427. DOI: 10.3892/mmr.2014.2863. 